COMBINATORICS AND GRAPH THEORY

BANACH CENTER PUBLICATIONS, VOLUME 25

PWN-POLISH SCIENTIFIC PUBLISHERS

WARSAW 1989

\title{
GRAPHS WHICH ARE LOCALLY PATHS
}

\author{
T. D. PARSONS \\ The late Professor of the California State University at Chico \\ Chico, California, U.S.A. \\ TOMAŽ PISANSKI \\ California State University at Chico \\ Chico, California, U.S.A \\ and \\ University of Ljubljana \\ Ljubljana, Slovenia, Yugoslavia
}

Graphs that are locally paths and cycles are studied. In particular, circulant graphs that are locally paths are determined.

\section{Graphs with prescribed links}

All graphs in this note are finite, simple, and connected unless specified otherwise.

Let $F$ be a family of isomorphism classes of graphs. A graph $G$ is said to be an $F$-graph if for each vertex $v$ of $G$ the link graph $\operatorname{Link}(v)$, i.e. the graph induced on the set of neighbors of $v$, belongs to $F$. Let $\operatorname{Link}(G)$ denote the family of isomorphism classes of all $\operatorname{Link}(v)$, where $v$ is a vertex of $G$. If $\operatorname{Link}(G)$ consists of a single graph, say $H$, we simply write $\operatorname{Link}(G)=H$ and say that $G$ is a constant link graph. In such a case $G$ is regular of valence $|V(H)|$. The problem of existence of $F$-graphs for a given family $F$ is hopelessly difficult in general since it is a generalization of Zykov's problem [Z] which is itself recursively unsolvable. However, for special families $F$ of graphs it can be quite simple-locally complete graphs are clearly complete. For certain families $F$ it becomes quite an interesting problem. If $F$ consists of all Hamiltonian graphs

Work supported in part by grants from the California State University, Research Council of Slovenia, Yugoslavia, and by the NSF (Grant DMS-8717441). 
we deal with the so-called locally Hamiltonian graphs that were first studied by Z. Skupień [Sk1, Sk2, Sk3]; see also [PS1, PS2]. It seems that these were chronologically the first examples of $F$-graphs.

In this note we consider a special family of links. Let $P$ denote the family of all paths $P=\left\{P_{n}: n>1\right\}$ and let $C$ denote the family of all cycles $C=\left\{C_{n}: n>2\right\}$. Let $P C$ denote the union of $P$ and $C$. Finally, for a given family $F$ let $F^{\prime}$ denote the family of isomorphism classes of graphs that we obtain from $F$ by taking finite (vertex disjoint, repeated) unions of elements from $F$. This means that $F^{\prime}$ contains disconnected graphs with components from $F$. First we mention briefly $P C$-graphs.

\section{2. $P C$-Graphs}

In this section we give a topological characterization of $P C$-graphs. Let $G$ be a $P C$-graph. Then each vertex is either a $P$-vertex (its link is a path) or it is a $C$-vertex (its link is a cycle). A cycle of length 3 is called a triangle. The proof of the following result is easy and is omitted.

Proposition 2.1. In a PC-graph each edge belongs to at least one triangle and to at most two triangles.

According to this proposition there is a natural partition of edges of a $P C$-graph into two classes. Edges belonging to two triangles will be called inner edges and edges that belong to a single triangle will be called boundary edges. Clearly all edges incident to a $C$-vertex are inner edges. On the other hand, each $P$-vertex has two incident boundary edges. That is why we call $C$-vertices also inner vertices and we call $P$-vertices also boundary vertices. Finally, we will call a triangle an inner triangle if all three of its sides are inner edges, i.e. if it is adjacent to three other triangles; otherwise it is called a boundary triangle. A boundary triangle may have 1,2, or 3 boundary sides. If it has 3 boundary sides we get $K_{3}$ which is an exception among $P C$-graphs. If a boundary triangle has 2 boundary sides then there is a $P_{2}$-vertex present in a $P C$-graph. Such a triangle is called a pending triangle and a $P_{2}$-vertex is called a pending vertex. It is always possible to remove a pending vertex and thereby a pending triangle and the resulting graph remains a $P C$-graph.

Proposition 2.2. A PC-graph different from $K_{3}$ has a $P_{2}$-vertex if and only if it has a pending triangle.

Proof. A $P_{2}$-vertex is incident to exactly two boundary edges and a single triangle. If the third side of this triangle is a boundary edge, then the graph is $K_{3}$. Otherwise we are dealing with a pending triangle. On the other hand, it is clear that a pending triangle gives rise to a $\boldsymbol{P}_{2}$-vertex, i.e. the vertex incident with the two boundary edges. 
Proposition 2.3. $G$ is a maximal outer planar graph if and only if $G$ is a P-graph which can be reduced by removal of a sequence of pending triangles to the graph $K_{3}$.

Proof. By induction; see also Brown and Connelly [BC2, p. 200].

This means that by removal of a sequence of pending triangles we eventually arrive at a $P C$-graph that is either $K_{3}$ or has no $P_{2}$-vertex. The reverse operation is attaching pending triangles to boundary edges. It also yields a $P C$-graph if we start from a $P C$-graph. Moreover, the $C$-vertices are not changed. The resulting graph remains a $P C$-graph. From now on we will disallow $P_{2}$-vertices in a $P C$-graph.

Proposition 2.4. The boundary edges and boundary vertices of a PC-graph $G$ form a graph $\partial G$ which is a union of boundary cycles. Each boundary cycle has length $>3$.

Proof. Since a boundary edge lies in a single triangle both of its endpoints must be $P$-vertices = boundary vertices. Each $P$-vertex is incident with precisely two boundary edges. This means that $\partial G$ is a regular graph of degree 2 , therefore it is a union of cycles. A cycle of length 3 in $\partial G$ would imply that $G=K_{3}$, which is forbidden.

To each graph $G$ we may associate a simplicial complex $K(G)$ in which the simplices are complete subgraphs and incidence relation is the subgraph inclusion.

Proposition 2.5. A connected graph $G$ is a PC-graph if and only if there is a surface $S$, possibly with nonempty boundary $\partial S$, such that $K(G)$ is a triangulation of $S$ in which $\partial G=\partial S$; each triangle of $G$ is a face of the triangulation $K(G)$.

Proof. Let $T$ be a triangulation of $S$ and let $G(T)$ be its one-skeleton. Assume that each triangle of $G(T)$ is a face of $T$. This implies that each boundary vertex of $G(T)$ is a $P$-vertex and that each inner vertex is a $C$-vertex. In other words, $G(T)$ is a $P C$-graph.

Given a $P C$-graph $G$ we may construct a 2-cell embedding of $G$ into some closed surface $S^{\prime}$ by first specifying a local rotation $p_{v}$ for each vertex $v$ and then the switching $\lambda(e)$ for each edge $e$ of $G$, i.e. by defining an embedding scheme $(G, P, \lambda)$. Take an arbitrary vertex $v$ of $G$. Then Link $(v)$ is either a cycle or a path. In both cases the link induces a cyclic permutation $p_{v}$ of neighbors of $v$ (up to inversion). We may select any admissible rotation. Let $e=u v$ be an arbitrary edge of $G$. We will select $\lambda(e)$ in such a way as to make all triangles of $G$ the faces of the embedding. If $p_{v}(u)=p_{u}(v)$ or if $p_{v}^{-1}(u)=p_{u}^{-1}(v)$ then let $\lambda(e)=-1$, otherwise let $\lambda(e)=1$. By the theory of Stahl [St] this defines a 2-cell embedding of $G$ into some surface $S^{\prime}$. Now delete all nontriangular 
(open) 2-cells of this embedding. The graph $G$ is then embedded into some complex $T$, homeomorphic to some topological space $S$. By Proposition 2.4, $\partial G$ is a union of cycles. It is clear that the deleted 2-cells give rise to $\partial G$ and $S$ is therefore a surface with $k$ boundary components where $k$ is the number of cycles in $\partial G$.

COROLlARY 2.6. If $G$ is a P-graph then all vertices of $G$ lie on the boundary of $K(G)$.

COROLlary 2.7. If $G$ is a $C$-graph then $\partial S=\emptyset$.

Here we only briefly mention an analogous characterization of $(P C)^{\prime}$ graphs. The proofs will be given elsewhere. A vertex of a $(P C)^{\prime}$-graph with a disconnected link is called a singular vertex as opposed to a regular vertex whose link is connected. A (PC)'-graph with no singular vertex is obviously a $P C$-graph. Since Proposition 2.1 applies also to $(P C)^{\prime}$-graphs the definition of a boundary graph can be obviously extended to $(P C)^{\prime}$-graphs.

PRoposition 2.8. A connected graph is a (PC)'-graph if and only if there is a pseudosurface $S$, possibly with nonempty boundary $\partial S$, such that $K(G)$ is a triangulation of $S$ in which $\partial G=\partial S$; each triangle of $G$ is a face of the triangulation $K(G)$.

COROLLARY 2.9. If $G$ is a $P^{\prime}$-graph then all vertices of $G$ lie on the boundary of $K(G)$.

Corollary 2.10. If $G$ is a $C^{\prime}$-graph then $\partial S=\varnothing$.

Notice, however, that in general attaching disks in various ways to a pseudosurface with several boundary components may result in nonhomeomorphic pseudosurfaces. Now we return to the study of $P C$-graphs.

The topological operation of attaching or deleting disks to a surface has a combinatorial counterpart. An inner vertex $v$ of a $P C$-graph $G$ with the property that all vertices of Link $(v)$ are inner vertices will be called superfluous. If we delete an inner vertex which is not superfluous we would obtain a $(P C)^{\prime}$-graph with singular vertices. Therefore we forbid such a deletion. If we delete a superfluous vertex $v$ from $G$ we get a graph $G-v$ which is still a $P C$-graph. The reverse operation "fills in a hole" and adds a vertex. It diminishes the number of boundary cycles by one. By a sequence of vertex additions we obtain a (uniquely determined) graph $c(G)$ which we call a completion of $G$. Note that $c(G)$ is a $C$-graph.

Finally, let us mention the fact that regular $P$-graphs and regular $C$-graphs are constant link graphs. Here we propose an open problem.

Problem 2.11. For each surface (compact 2-manifold) $S$ determine all integers $k$ such that there is a regular $P$-graph $G$ whose completion $c(G)$ is a regular $k$-valent triangulation of $S$. 


\section{The Euler formula for $P C$-graphs}

In this section we prove a formula for $P C$-graphs that is derived from the Euler formula for 2-cell embeddings of graphs.

Let $G$ be a $P C$-graph. Let $p_{k}$ denote the number of vertices $v$ such that $\operatorname{Link}(v)=P_{k}$, let $c_{k}$ denote the number of vertices $v$ such that $\operatorname{Link}(v)=C_{k}$. Furthermore, let $p=p_{3}+p_{4}+\ldots$ and $c=c_{4}+c_{5}+\ldots$ Let $n=p+c$ be the number of vertices, $e$ the number of edges, and $t$ the number of triangles in $G$. Let $d$ be the number of boundary cycles in $\partial G$. Let $S$ be the surface determined by $K(G)$ and let $S$ ' be the surface which is obtained from $S$ by "filling in the $d$ holes". Let $\chi=\chi\left(S^{\prime}\right)$ be the corresponding Euler characteristic. Then we can prove the folowing result.

Proposition 3.1. For each PC-graph $G$

$$
\sum(k-6) c_{k}+\sum(k-4) p_{k}=6(d-\chi) \quad \text { and } \quad p \geqslant 4 d \text {. }
$$

Proof. We start with the Euler formula $\chi=n-e+f$, where $f=t+d$ is the number of faces of $S^{\prime}$. By counting the edges on faces we obtain $3 t+p=2 e$. By counting the degrees we get $\sum k\left(c_{k}+p_{k}\right)=2 e$. This means that we can express $n$, $e$, and $f$ in terms of $d, c_{k}, p_{k}$. This is how we obtain the formula. The inequality $p \geqslant 4 d$ follows from the fact that each boundary cycle is of length at least 4 .

If we select certain special subclasses of $P C$-graphs we get the following results.

Corollary 3.2. Let $G$ be a PC-graph.

(a) If $G$ is regular of valence $k$ then

$$
(k-6) c+(k-4) p=6(d-\chi)=(k-6) n+2 p .
$$

(b) If $G$ is a $C_{k}$-graph then $6 \chi=(6-k) n$.

(c) If $G$ is a $P_{k}$-graph then $6(d-\chi)=(k-4) n$, and $n \geqslant 4 d$.

\section{Circulant graphs that are locally paths}

In this section we extend Parsons' result from [P] to characterize connected $P_{k}$-circulant graphs. Let $B_{k}$ denote the set of all connected circulant graphs that are locally $P_{k}$-graphs. As in the previous paper [P] let $G(n, S)$ denote the circulant graph on $n$ vertices with symbol $S$.

THEOREM 4.1. $G(n, S)$ belongs to $B_{k}$ if and only if one of the following conditions holds.

(a) $n=2, k=1, S=\{1\}$ and $G(n, S)=K_{2}$ [Link $\left.=P_{1}\right]$.

(b) $n=3, k=2, S=\{-1,+1\}$ and $G(n, S)=K_{3}$ [Link $\left.=P_{2}\right]$.

(c) $n \geqslant 7, k=4, S=a\{-1,+1,-2,+2\}$ for some a from $Z_{n}^{*}$, the group of units of the ring $Z_{n}\left[\right.$ Link $\left.=P_{4}\right]$. 
Proof. It is easy to verify that the graphs specified in this theorem are indeed $P_{k}$-graphs. We have to prove that there are no other such graphs. The proof is divided into several disjoint cases.

Case 1: There exists an $a$ from $S$ such that $2 a=n$.

Case 1.1: $S=\{a\}$. Then $G(n, S)$ is connected if and only if $n=2$, $k=1, S=\{1\}$, and $G(n, S)=K_{2}$.

Case 1.2: There exists an element $b$ in $S$, different from $a$ and adjacent to $a$. This is impossible since we would get a triangle $C_{3}=(b$, $a, a-b)$

Case 2: For each $a$ from $S$ the corresponding $-a$ also belongs to $S$ but $a \neq-a$. Hence $|S|$ is even, say $|S|=2 m$, and $\operatorname{link}(0)=P_{2 m}$. The map $f: S \rightarrow S$ with $f(a)=-a$ induces an automorphism of $G(n, S)$ fixing 0 . It also induces a nontrivial automorphism of $P_{2 m}$. Since $\operatorname{Aut}\left(P_{2 m}\right)=Z_{2}$ the nontrivial automorphism is uniquely defined. Therefore $P_{2 m}$ is of the following form:

$$
P_{2 m}=\left(-x_{1},-x_{2}, \ldots,-x_{m}, x_{m}, \ldots, x_{2}, x_{1}\right),
$$

where $S=\left\{-x_{1},+x_{1},-x_{2},+x_{2}, \ldots,-x_{m},+x_{m}\right\}$. Therefore there exists an element $a$ in $S$ [with $-a$ also from $S$ ] such that $a$ is adjacent to $-a$ [and there exists an element $b$ in $S$ such that $b$ is not adjacent to $-b]$. Namely, $a=x_{m}$ and $b=x_{1}$. If $a$ is adjacent to $-a$ then $2 a$ belongs to $S$.

Case 2.1: $a, 2 a,-a,-2 a$ are distinct elements of $S$. Therefore Link $(0)$ contains $P_{4}=(-2 a,-a,+a,+2 a)$.

Case 2.1.1: $S=\{-a,+a,-2 a,+2 a\}$. This implies $k=4$. Since we must have $\operatorname{Link}(0)=P_{4}$ neither $3 a$ nor $4 a$ belongs to $S$. This implies $n \geqslant 7$ and $\operatorname{gcd}(n, a)=1$.

Case 2.1.2: $S$ contains a fifth element, say $b$. This would imply that $b$ is adjacent to $2 a$, which is impossible.

Case 2.2: Both $a$ and $2 a$ belong to $S$ and $2 a=-a$.

Case 2.2.1: $S=\{-a,+a\}$, and $2 a=-a$. Hence $n=3, k=2$, $S=\{-1,+1\}$, and $G(n, S)=K_{3}$.

Case 2.2.2: $S$ contains a third element, say $b$. We do not obtain anything new. The case reduces to 2.1 , in which $b$ plays the role of $a$.

These cases cover all possibilities and thus the theorem is proved.

It follows from the proof that for each $n$ we obtain at most one circulant graph that is locally $P_{k}$. Let $\operatorname{Circ}(n)$ denote this graph. The following results are given here (without proof) for the sake of completeness.

Proposition 4.2. Circ(2) is not a triangulation. Circ(3) $=K_{3}$ is a triangulation of a disk, $\operatorname{Circ}(2 m), m \geqslant 4$, is a triangulation of a cylinder [the antiprism], and $\operatorname{Circ}(2 m-1), m \geqslant 4$, is a triangulation of a Möbius strip. All triangulations have only boundary vertices. 
Proposition 4.3. Aut $(\operatorname{Circ}(2))=Z_{2}$, Aut $(\operatorname{Circ}(3))=S_{3}$, and $\operatorname{Aut}(\operatorname{Circ}(n))$ $=D_{n}, n \geqslant 7$, where $Z_{n}, S_{n}$, and $D_{n}$, denote the cyclic, symmetric, and dihedral group of respective orders $n, n$ !, and $2 n$.

\section{Characterization of $P_{k}$-graphs, for $k \leqslant 5$}

THEOREM 5.1. The only connected $P_{k}$-graphs, $k \leqslant 4$, are the ones given in Theorem 4.1.

Proof. For $k \leqslant 2$ the result is obvious. For $k=3$ there are no such graphs, see $[\mathrm{BCl}]$. Now we prove it for $k=4$. By Corollary 3.2 (c) we have $6(d-\chi)$ $=(k-4) n=0, n \geqslant 4 d$. It follows that the Euler characteristic equals the number of boundary components: $\chi=d$. For the sphere we get two boundary components. We are dealing with the cylinder. Also, for the projective plane the corresponding surface with boundary is the Möbius strip. It is then an easy verification that the only $P_{4}$-triangulations of the cylinder and the Möbius strip are indeed $\operatorname{Circ}(n), n \geqslant 7$.

Let us introduce the so-called T-construction. We start with an oriented 2-cell embedding of a simplicial cubic graph $K$ into an orientable surface $S$ such that no face of the embedding is a triangle. Next we form another graph $T(K)$ as follows. For each vertex $w$ of $K$ we form three vertices of $T(K)$. Hence $|V(T(K))|=3|V(K)|$. We would like $T(K)$ to be a regular 5-valent graph. We will construct three types of edges in $T(K)$ : boundary edges, triangle edges and diagonal edges. Each vertex of $T(K)$ will be the endvertex of two boundary edges, two triangle edges and one diagonal edge.

Let $u$ be an arbitrary vertex of $K$. Let $e, f$, and $g$ be the three edges of $K$ incident with $u$. Then we denote the three vertices of $T(K)$ corresponding to $u$ by $u_{e}, u_{f}$, and $u_{g}$. Let $v$ be the other endvertex of $e: e=u v$, and let $e, h$, and $i$ be the three edges incident with $v$. In general the edge $e$ lies on two faces of the embedding of $K$. Without loss of generality we may assume that feh... is a part of the oriented boundary of one of the two faces determined by $e$. If we have a coherent orientation of faces then ieg... is a part of the oriented boundary of the other face.

Now we can describe the adjacencies of a typical vertex, say $u_{f}$, of $T(K)$. The vertex $u_{f}$ is adjacent to $u_{c}$ and $u_{q}$. The three vertices of $T(K)$ belonging to the same vertex $u$ of $K$ form a triangle. Its edges are the triangle edges of $T(K)$.

Since we would like $T(K)$ to be a regular 5-valent graph we need three more adjacencies for $u_{f}$. We let $u_{f}$ be adjacent to $v_{h}$. We can explain this - adjacency by following the boundary ieg... There $h$ and $f$ are two consecutive edges incident with the 2-cell. By the same argument $u_{f}$ is adjacent to another vertex, say $w_{j}$, if $w$ represents the other endvertex of the edge $g$ and $j$ the next edge perpendicular to this face. Also, by the same argument $u_{g}$ is adjacent to $v_{i}$. This is how we construct the boundary edges of $T(K)$. 
So far we have 4 neighbors of $u_{f}$. The first two, namely $u_{e}$ and $u_{g}$, do not depend on the embedding of $K$. The edges involved are the triangular edges. The second pair, i.e. $v_{h}$ and $w_{j}$, is determined from the face structure of the embedding. The edges involved are the boundary edges. However, no orientability is needed in their description.

The last adjacency uses the orientation of $f e h . .$. and ieg... Namely we let $u_{f}$ be adjacent to $v_{i}$ since both $f$ and $i$ precede $e$ on the two oriented boundaries. By the same argument $u_{e}$ is adjacent to $w_{j}$. These edges are called the diagonal edges. This now determines a 5-regular graph $T(K)$.

The T-construction is of interest because of the following result.

THEOREM 5.2. The only connected $P_{5}$-graphs are the ones that can be obtained from an oriented embedding without triangular faces of a simplicial cubic connected graph with a T-construction.

Proof (sketch). From the construction it immediately follows that $T(K)$ is indeed a $P_{5}$-graph. The proof in the reverse direction is a little more involved. Let $G$ be a $P_{5}$-graph. We first identify the boundary edges. There are two boundary edges incident with a given vertex. It is easy to see that from the remaining three edges incident with a given vertex two lie on an inner triangle and the third one has to be a diagonal edge. In order to carry out this identification globally we have to have an orientable surface with boundary determined by $G$.

Compare [Ha] where a vague description of the $T$-construction is mentioned. There it is also possible to find a different proof of Theorem 5.1.

COROLlary 5.3. The surface with boundary that is determined by a connected $P_{5}$-graph is orientable.

Let us conclude with a couple of problems.

Problem 5.4. Give a characterization of $P_{k}$-graphs, for $k>5$.

We have seen that $P_{5}$-graphs cannot triangulate nonorientable surfaces. So the following problem is related to Problem 2.11.

Problem 5.5. For a given $k$ determine the surfaces that can be triangulated by a $\boldsymbol{P}_{\boldsymbol{k}}$-graph.

Acknowledgements. Great help and patience of the referee and Professor Z. Skupień are sincerely acknowledged. 


\section{References}

[BHT] G. S. Bloom, P. Hell and H. Taylor, Collecting autographs: n-node graphs that have n-integer signatures, in: Ann. New York Acad. Sci. 319 (1979), 93-102.

[BC1] M. Brown and R. Connelly, On graphs with a constant link, in: New Directions in the Theory of Graphs, F. Harary (ed.), Academic Press, New York 1973, 19-51.

[BC2] -, -, On graphs with a constant link, II, Discrete Math. 11 (1975), 199-232.

[Ha] J. I. H all, Graphs with constant link and small degree or order, J. Graph Theory 9 (1985), 419-444.

[He] P. Hell, Graphs with given neighborhoods I, in: Problèmes combinatoires et théorie des graphes (Orsay 1976), Colloq. Internat. CNRS 260 (1978), 219-223.

[HLW] P. Hell, H. Levinson and M. Watkins, Some remarks on transitive realizations of graphs, in: Proc. 2nd Caribbean Conf. Combinatorics Comput., R. C. and C. C. Cadogan (eds.), Barbados 1977, 115-122.

[PS1] C. M. Pareek and Z. Skupień, On minimal non-Hamiltonian locally Hamiltonian graphs (abstract), in: Ann. Discrete Math. 8 (1980), 265.

[PS2] -, -, On the smallest nonhamiltonian locally Hamiltonian graphs, J. Univ. Kuwait Sci. 10 (1983), 9-17.

[P] T. D. Parsons, Circulant graph imbeddings, J. Combin. Theory Ser. B 29 (1980), 310-320.

[Sk1] Z. Sku pień, Locally Hamiltonian graphs and Kuratowski theorem, Bull. Acad. Polon. Sci. Sér. Sci. Math. Astronom. Phys. 13 (1965), 615-619.

[Sk2] -, On the locally Hamiltonian graphs and Kuratowski's theorem, Prace Mat. 11 (1968), 255-264.

[Sk3] -, On generalizations of locally Hamiltonian graphs, in: Graphs, Hypergraphs and Block Systems, Proc. Sympos. Zielona Góra 1976, M. Borowiecki, Z. Skupień and L. Szamkołowicz (eds.), Zielona Góra 1976, 263-271.

[St] S. Stahl, Generalized embedding schemes, J. Graph Theory 2 (1978), 41-52.

[Z] A. A. Zykov, Problem 30, in: Theory of Graphs and Its Applications, Proc. Sympos. Smolenice 1963, Publishing House of the Czechoslovak Acad. Sci., Prague 1964, 164-165.

Presented to the Semester

Combinatorics and Graph Theory

September 14 - December 17, 1987 